\title{
Cervical Facet Fracture Dislocations-Management Algorithm and Tips for Reduction
}

\author{
Chaitanya Chikhale*, Mohammed Faizan and Sushil Mankar \\ NKP Salve Institute of Medical Sciences, India
}

Submission: August 15, 2017; Published: August 29, 2017

*Corresponding author: Chaitanya Chikhale, NKP Salve Institute of Medical Sciences, Nagpur, Maharashtra, India-440019, Email: chaitanya14486@gmail.com

\section{Short Communication}

Cervical facet fractures and dislocations are osteoligamentous injuries of the cervical spine. These are a type of flexion distraction injuries as per the Allen Ferguson classification of sub axial cervical spine injuries. These injuries can be simplified into Uni-facetal dislocations, Bi-facetal dislocations or Facet fracture with a combination of above injuries also possible [1].

These patients typically present with a history of fall from height or a high velocity trauma or even sports injuries like in contact sports is also possible. Clinically the diagnosis in a conscious patient can be obtained from the history of mechanism of injury, the attitude of the neck would be stiff and the rotations and flexion extension of the sub axial cervical spine would be restricted. The patient might also complain of radicular pain in his upper limbs or in most cases there is neurological deficit particularly if the injury was a high impact one.

However, in many cases there is no obvious neurological deficit particularly the ones who had a facet fracture along with a dislocation as it tends to increase the canal diameter and the cervical spinal cord may escape unharmed [2].

Such patients when they present to the emergency department should be immobilised properly following the ATLS protocols. Radiological investigations like xrays of the cervical spine AP and lateral views should be done. It is not uncommon to miss these injuries at primary presentation and such patients then land up with neglected traumatic facet dislocations. Dynamic xrays (flexion /extension views) may be indicated in certain cases where the instability is clinically suspected but not obvious on plain xrays, but must always be done under supervision.

Lateral xray of cervical spine should be assessed for loss of disc height indicating a probable herniation and for the amount of listhesis. Usually less than $25 \%$ listhesis of upper vertebra on the lower level is indicative of unilateral dislocations and $50 \%$ or above amount of listhesis is usually bilateral. AP xrays likewise can tell us about any rotator abnormality involved along with subluxation or dislocation by looking at the alignment of spinous processes.
CT scans should be done to look for bony anatomy ,alignment, side of the facet dislocations in case of unilateral dislocations and fractures whereas MRI is indicated if clinically a disc herniation is suspected at the affected level or the patient is having neurological deficit or if the deficit has progressed gradually particularly while attempting reduction. It can also give us an idea about the prognosis of neurological recovery apart from planning the surgical approach [3].

Facet fractures and subluxations without significant displacement and no neurological deficit can be managed conservatively in a cervical orthosis for 6-12 weeks. Most however require some intervention. It is a common practise to attempt reduction of the dislocation particulary if the patient is conscious. An emergent closed reduction should however be avoided in a patient whose consciousness is altered. Application of skull traction with Gardener Well Tongs with attempt at reduction may be done either in the casualty itself or under $\mathrm{C}$ ARM guidance in the operation theatre.

For closed reduction of the dislocation a gradually increasing traction is applied and the neck is kept in flexion, serial lateral xrays are done to watch for the facets to unlock, once the dislocation starts to reduce as seen by parallel orientation of facets on xrays then the traction is continued in gentle extension of the neck. In case of unilateral dislocations traction is applied and the neck is rotated to the side of facet dislocation in flexion followed by gently bringing the neck back to midline while extending it gradually.

It is imperative to watch for deterioration of neurology of the patient during the reduction manoeuvres as in many cases there could be a anterior disc herniation which may impinge on the cord during the closed reduction. However if the patient already has a partial or complete neurological deficit then in our experience closed reduction should be attempted irrespective of the status of disc as we have not seen further deterioration of neurology in our practise in such cases [4].

For those patients who fail closed reduction or develop neuro-deficit during reduction or have progressive neurological 


\section{Open Access Journal of Neurology \& Neurosurgery}

deficit are candidates for open reduction. If the injury is fresh then we prefer to use an anterior approach. A standard left sided Smith -Robinson approach is used, we prefer to use handheld retractors over self retaining ones carefully protecting the carotid vessels laterally and the oesophagus medially, once the disc is identified and level confirmed we perform a discectomy and try to remove as much disc as possible ventrally till PLL is seen and laterally till the limits of unco-vertebral joints. Then reduction is attempted by first placing the tip of a forearm bone lever in the middle of lower vertebral endplate to disengage the facets while simultaneously applying downward pressure on the upper vertebral body by your thumb. A clunk is felt once the reduction is achieved [5].

In case of unilateral facet dislocations once the discectomy is done and the Caspar pins are in place, the upper vertebral pin is then rotated towards the side of facet dislocation and reduction is obtained [6].

Sometimes reduction is not possible by anterior approach alone particularly cases with delayed presentation, in such cases a first stage posterior release is done by removing the part of facet and reduction obtained by traction followed by flexion followed by gentle extension of neck and the reduction is then held by applying lateral mass screws at two levels. Following this the patient is turned supine and anterior cervical discectomy and fusion by using an interbody cage or iliac crest bone graft and plating is done. Some authors have also used an anterior followed by posterior approach or a posterior only approach $[7,8]$.

Conclusion-Management of cervical facet fracture dislocations requires adherence to an algorithmic approach as discussed above. Closed reduction should always be attempted in a conscious patient under clinic radiological supervision. Anterior cervical discectomy and fusion alone, gives satisfactory reduction in most cases of reducible cervical facet fracture dislocations. Combined approach should be reserved for cases who failed open anterior reduction.

A case of 55 yrs old man with history of fall from height presented with quadriparesis and bowel bladder involvement was operated with anterior cervical discectomy.

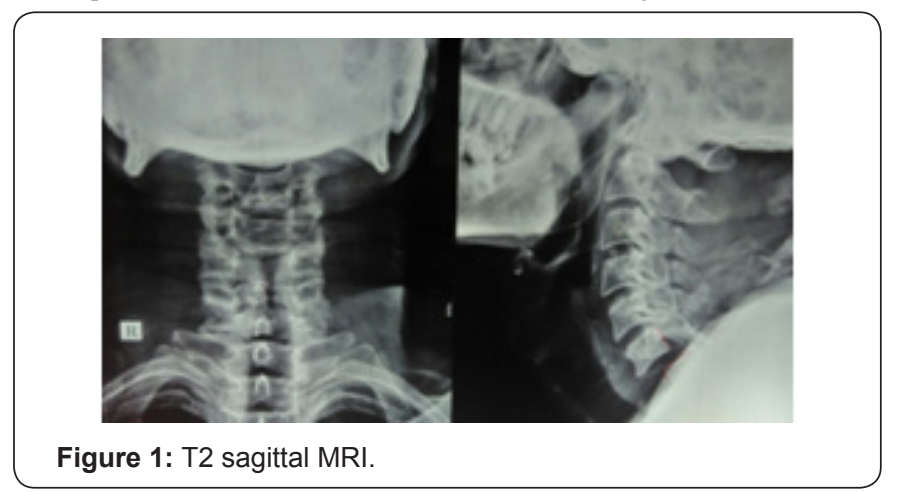

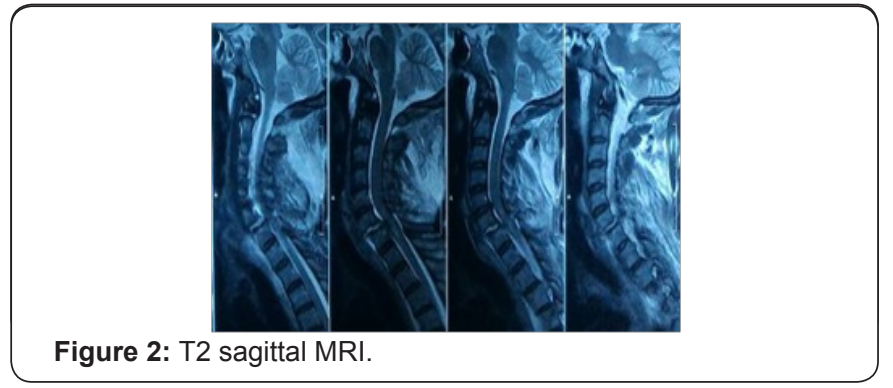

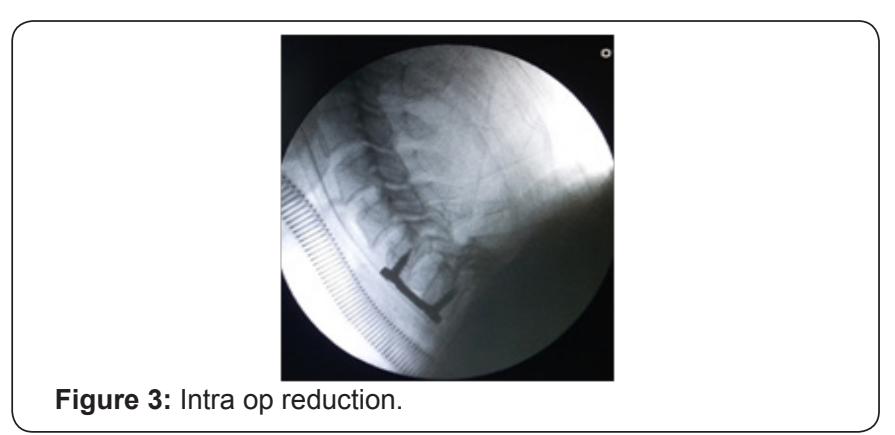

Figure 3: Intra op reduction.

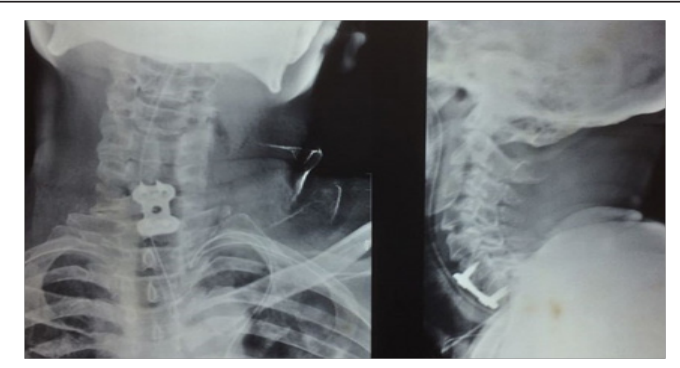

Figure 4: Post op xray.

\section{Referances}

1. Hadley MN, Fitzpatrick BC, Sonntag VK, Browner CM (1992) Facet fracture-dislocation injuries of the cervical spine. Neurosurgery 30(5): 661-666.

2. Mahale YJ, Silver JR (1992) Progressive paralysis after bilateral facet dislocation of the cervical spine. J Bone Joint Surg Br 74(2): 219-223.

3. Joaquim AF, Patel AA (2013) Subaxial cervical spine trauma: evaluation and surgical decision-making. Global Spine J 4(1): 63-70.

4. Srivastava SK, Aggarwal RA, Bhosale SK, Nemade PS (2016) Neglected dislocation in sub-axial cervical spine: Case series and a suggested treatment protocol. J Craniovertebr Junction Spine 7(3): 140-145.

5. Ordonez BJ, Benzel EC, Naderi S, Weller SJ (2000) Cervical facet dislocation: techniques for ventral reduction and stabilization. J Neurosurg 92(1): 18-23.

6. Andreshak JL, Dekutoski MB (1997) Management of unilateral facet dislocations: a review of the literature. Orthopedics 20(10): 917-926.

7. Song KJ, Lee KB (2008) Anterior versus combined anterior and posterior fixation/fusion in the treatment of distraction-flexion injury in the lower cervical spine. J Clin Neurosci 15(1): 36-42.

8. Abumi K, Shono Y, Kotani Y, Kaneda K (2000) Indirect posterior reduction and fusion of the traumatic herniated disc by using a cervical pedicle screw system. J Neurosurg 92(1): 30-37. 
This work is licensed under Creative Commons Attribution 4.0 Licens DOI: 10.19080/OAJNN.2017.5.555669
Your next submission with Juniper Publishers will reach you the below assets

- Quality Editorial service

- Swift Peer Review

- Reprints availability

- E-prints Service

- Manuscript Podcast for convenient understanding

- Global attainment for your research

- Manuscript accessibility in different formats ( Pdf, E-pub, Full Text, Audio)

- Unceasing customer service

Track the below URL for one-step submission https://juniperpublishers.com/online-submission.php 